\title{
UCRL-JRNL-230853
}

LAW RENCE LIVERMORE N A T IO N A L LABORATORY

The Zeta Potential of

Surface-Functionalized Metallic Nanorod Particles in Aqueous Solution

G. M. Dougherty, K. A. Rose, J. B.-H. Tok, S. S. Pannu, F. Y. S. Chuang, M. Y. Sha, G. Chakarova, S. G. Penn

May 10, 2007

Electrophoresis 
This document was prepared as an account of work sponsored by an agency of the United States Government. Neither the United States Government nor the University of California nor any of their employees, makes any warranty, express or implied, or assumes any legal liability or responsibility for the accuracy, completeness, or usefulness of any information, apparatus, product, or process disclosed, or represents that its use would not infringe privately owned rights. Reference herein to any specific commercial product, process, or service by trade name, trademark, manufacturer, or otherwise, does not necessarily constitute or imply its endorsement, recommendation, or favoring by the United States Government or the University of California. The views and opinions of authors expressed herein do not necessarily state or reflect those of the United States Government or the University of California, and shall not be used for advertising or product endorsement purposes. 


\section{The Zeta Potential of Surface-Functionalized Metallic Nanorod Particles in Aqueous Solution}

George M. Dougherty ${ }^{1}$, Klint A. Rose ${ }^{1}$, Jeffrey B.-H. Tok ${ }^{1}$, Satinderpall S. Pannu ${ }^{1}$, Frank Y. S. Chuang ${ }^{2}$, Michael Y. Sha ${ }^{3}$, Gabriela Chakarova ${ }^{3}$, Sharron G. Penn ${ }^{3}$

${ }^{1}$ Lawrence Livermore National Laboratory, Livermore, CA, USA

${ }^{2}$ University of California-Davis Cancer Center, Davis, CA, USA

${ }^{3}$ Oxonica, Mountain View, CA, USA

Author Contact Information:

Dr. Klint A. Rose

7000 East Avenue, L-223

Lawrence Livermore National Laboratory

Livermore, CA 94551

rose38@llnl.gov

phone (925) 423-1926

fax (925) 422-2783

Keywords: nanoparticle, nanorod, coating, zeta potential, aqueous 


\begin{abstract}
Metallic nanoparticles suspended in aqueous solutions, and functionalized with chemical and biological surface coatings, are important elements in basic and applied nanoscience research. Many applications require an understanding of the electrokinetic or colloidal properties of such particles. In this paper we describe the results of experiments to measure the zeta potential of metallic nanorod particles in aqueous saline solutions, including the effects of $\mathrm{pH}$, ionic strength, metallic composition, and surface functionalization state. Particle substrates tested include gold, silver, and palladium monometallic particles as well as gold/silver bimetallic particles. Surface functionalization conditions included 11-mercaptoundecanoic acid (MUA), mercaptoethanol (ME), and mercaptoethanesulfonic acid (MESA) self-assembled monolayers (SAMs), as well as MUA layers subsequently derivatized with proteins. Zeta potential data for typical charge-stabilized polystyrene particles are also presented for comparison. Experimental data are compared with theory. The results of these studies are useful in predicting and controlling the aggregation, adhesion, and transport of functionalized metallic nanoparticles within microfluidic devices and other systems.
\end{abstract}




\section{Introduction}

\subsection{Nanoparticles and electrophoretic transport}

There is broad interest in using nanoparticles in aqueous solution as substrates for the attachment of various chemical and biochemical species. Covalently functionalized metallic particles, such as colloidal gold, have made possible a range of new methods in the detection of DNA and protein targets [1-6], surface plasmon resonance and surface enhanced Raman spectroscopy [7-12], cellular and sub-cellular targeting for imaging and therapeutics [13-15], and the formation of higher-order nanostructures and nanomaterials by self-assembly [16-19], among other applications. Recent work has focused on the enhanced capabilities provided by engineered nanorod (or nanowire) particles [20-25]. The employment of metallic particles in aqueous solution requires the control of aggregation, adhesion, and transport of the particles. These issues are of particular importance when the particles are used within microfluidic lab-on-a-chip systems and other high surface-area environments [26].

We are investigating the use of encoded metallic nanorod particles (Nanobarcodes ${ }^{\circledR}$ particles, Oxonica, Mountain View, CA) $[20,22,23]$ as solution array elements for use in integrated multiplex biodetection systems. The particles have a nominal diameter of $250 \mathrm{~nm}$ and length of $6 \mu \mathrm{m}$, and are composed of alternating segments of dissimilar metals, as shown in Fig. 1. In this application, the particles are functionalized with selfassembled monolayers and affinity proteins. In order to understand the adhesion, 
aggregation, and transport behavior of the particles, as well as their compatibility with other materials such as glass and polydimethylsiloxane (PDMS) used in the fabrication of microfluidic devices, it is desirable to have data on the electrokinetic properties of these particles in aqueous solution. The present study was undertaken in order to obtain data on the variation of zeta potential with respect to the $\mathrm{pH}$ and ionic strength of the solution, and the material and surface functionalization state of the particles.

The zeta potential, the effective surface potential at the hydrodynamic "shear surface" close to the solid-liquid interface, is a key parameter governing the electrokinetic behavior of particles in solution. This parameter determines the electrophoretic mobility of the particles within an external electric field, as well as the electrostatic repulsion between particles (or between a particle and a bounding surface) that acts to prevent or promote particle attraction and adhesion. The interaction between small colloidal particles in solution is described by the DLVO model $[27,28]$, where the total energy as a function of separation distance is the sum of a long range electrostatic repulsive contribution, and a short range attractive contribution due to Van der Waals interactions. Large zeta potentials of like sign maximize the electrostatic repulsive force and therefore minimize aggregation.

Because of the close relationship between zeta potential and electrophoretic mobility, measurements of zeta potential may be performed by imposing a known electric field across a suspension of particles, measuring the resulting electrophoretic velocity of the particles, and then calculating the zeta potential. The electrophoretic velocity observed in 
such an experiment is equal to the applied field multiplied by the electrophoretic mobility $v_{E}:$

$$
\bar{u}_{E}=v_{E} \cdot \bar{E}
$$

The electrophoretic mobility is related to the zeta potential $\zeta$ by the HelmholtzSmoluchowski equation $[29,30]$

$$
v_{E}=\frac{\varepsilon_{r} \varepsilon_{0} \zeta}{\eta}
$$

In this expression $\varepsilon_{0}$ is the dielectric permittivity of free space, $\varepsilon_{r}$ is the dielectric constant of the medium, and $\eta$ the fluid viscosity. This equation holds for the case where the ionic double layer thickness is much less than the particle radius, that is, where $\kappa a>>1$, where $a$ is the particle radius and $1 / \kappa$ is the thickness of the double layer, a function of the ionic strength of the suspending medium [30]:

$$
\kappa=\left(\frac{2 e^{2} z^{2} n}{\varepsilon_{0} \varepsilon_{r} k T}\right)^{\frac{1}{2}}
$$

Here, $e$ is the charge on an electron, $n$ the concentration of ions in ions $/ \mathrm{m}^{3}, z$ the net ionization of the ions, $k$ the Boltzmann constant and, $T$ is temperature. For the particles 
and solutions in this study, $\kappa a$ varies from 42 to 130, so the Helmholtz-Smoluchowski equation offers a good approximation. While the equation was originally derived for the case of a spherical insulating particle, it has subsequently been shown to also apply to our particular case of a nonspherical conducting particle, assuming $(1 / \kappa a) \exp \left(z e\left(\zeta+E_{\infty} c\right) / 2 k T\right)<<1$ where $E_{\infty}$ is the applied field and $c$ is the particle halflength $[31,32]$. The conducting nanorod particles exhibit increased alignment versus dielectric particles in an applied electric field, but this alignment does not affect the translational motion of the particles [Rose].

\subsection{Zeta potential theory}

Theoretical models are available to predict the zeta potential of particles as a function of the surface properties (e.g., surface functionalization, and striping pattern) and solution properties. We briefly describe the relevant models for bare metal particles, particles with a self-assembled monolayer (SAM) coating or with bound proteins, and particles with stripes of different materials along their lengths. In this paper we compare the predictions for SAM coated and striped particles to experimental results. In all cases we assume the voltage drop across a particle, $V_{p} \approx E_{\propto} c$, is well below the 1 to $2 \mathrm{~V}$ threshold necessary to generate electrochemical reactions at the surface.

To model the zeta potential we divide the problem into three regions starting at the surface. For a bare particle this describes the actual surface of the particle. For a coated particle, we define the surface as the region at the boundary between the fluid and the 
coating. Adsorbed to the surface is a layer of ions referred to as the Stern layer or inner Helmholtz plane (IHP). Ions outside the Stern layer are diffuse and the boundary between this diffuse layer and the adsorbed layer is the outer Helmholtz plane. We define potentials, $\psi_{o}, \psi_{i}, \psi_{d}$ and charge densities $\sigma_{o}, \sigma_{i}, \sigma_{d}$, at the surface and inner and outer Helmholtz planes, respectively. To achieve electroneutrality, the charge in the IHP and OHP must fully shield the surface charge, such that $\sigma_{o}+\sigma_{i}+\sigma_{d}=0$. For a symmetric, 1-1 electrolyte, the charge density at the OHP is related to the potential at this plane by the Grahame equation,

$$
\sigma_{d}=\frac{4 n z e}{\kappa} \sinh \left(\frac{z e \psi_{d}}{2 k T}\right)
$$

where $\kappa$ is defined in Eq. (3). The potential, $\psi_{d}$, is approximated as the zeta potential, $\zeta$ [Schweiss].

\subsubsection{Bare metal particles}

For bare metal particles without an oxide layer, we assume the surface has no inherent charge, i.e. $\sigma_{o}=0$. The negative zeta potential is instead due to preferential adsorption of negative ions to the surface; as Hunter [Hunter] explains, this preferential adsorption may be due to anions not hydrating as readily as cations and therefore adsorbing more readily to the surface. Assuming the adsorption is confined to anions only, the charge density at the IHP is calculated using a Langmuir isotherm and defined as 


$$
\sigma_{i}=z_{-} e N X_{S} \exp \left[-\left(z_{-} e \psi_{i}+\theta_{-}\right) / k T\right]
$$

where $N$ is the number of adsorption sites, $x_{s}$ is the molar fraction of the bulk electrolyte, z. is the valence of the anion(s), and $\theta$ - is the adsorption potential from the. The IHP potential is related to the OHP potential through the capacitance per unit area, $C_{i n}$, across this layer, such that $\psi_{i}=\psi_{d}+\sigma_{d} / C_{i n}$. Assuming no surface charge, equations (4) and (5) are equated as $\sigma_{i}=-\sigma_{d}$ to produce the following relation:

$$
\sigma_{d}(\zeta, n)=-z_{-} e N x_{s} \exp \left[-\left(\frac{z_{-} e}{k T}\left(\zeta-\frac{\sigma_{d}(\zeta, n)}{C_{i n}}\right)+\frac{\theta_{-}}{k T}\right)\right] .
$$

Eq. (6) can be solved numerically and fit to zeta potential data to determine the parameters, $N, C_{i n}$, and $\theta$. The number of adsorption sites, $N$, is typically $10^{14}$ to $10^{15}$ sites $/ \mathrm{cm}^{2}$, the capacitance is generally between 16 and $30 \mu \mathrm{F} / \mathrm{cm}^{2}$, and $\theta$ is 1 to 5 times kT [Hunter].

\subsubsection{Coated particles}

Two distinct approaches are taken to describe the zeta potential for a polymer SAM coated particle as a function of $\mathrm{pH}$. Ohshima et al. developed an equation for the mobility of a polymer coated particle taking into account the polymer length, flow penetration into the polymer layer, surface coverage, and the underlying zeta potential of the uncoated particle [Ohshima, Rodriguez]. They extended this work to particles with antigen and antibody coatings, solving for the relevant parameters using data from latex 
particles [Nakamura, Rodriguez]. While this model includes most of the electrostatic and hydrodynamic effects of the polymer layer, it is difficult to fit to experimental data for the case of an underlying zeta potential on the particle. We therefore use the one-site dissociation model, described for SAMs by Schweiss et al [Schweiss]. This approach ignores the underlying zeta potential, and assumes the charge at the interface between the polymer and solution is due only to association/dissociation kinetics of the polymer.

Using the one-site dissociation model, the charge at the surface (defined in this case as the polymer/fluid interface) is

$$
\sigma_{o}=\frac{-e N_{s}}{1+10^{(p K a-p H)} \exp \left(-\frac{e \psi_{o}}{k T}\right)}
$$

where $N_{s}$ is the total number of surface groups, pKa is the acid dissociation constant for the polymer, and $\psi_{o}$ is the potential at the polymer surface [Hunter]. We assume in this case that there are no adsorbed ions in the IHP so that $\sigma_{o}=-\sigma_{d}$ and $\psi_{o}=\psi_{d}+\sigma_{d} / K$, where $K$ is the capacitance/area between the surface of the polymer and the OHP. The resulting equation,

$$
\sigma_{d}+\frac{e N_{s}}{1+10^{(p K a-p H)} \exp \left(-\frac{e}{k T}\left(\zeta-\frac{\sigma_{d}}{K}\right)\right)}=0
$$

is fit to experimental zeta potential data to determine the values for the parameters, $N_{s}$, pKa, and $K$. 


\subsubsection{Striped particles}

The mobility of a nanorod with stripes of dissimilar metals along its length can be predicted using zeta potential data or models for homogenous particles under the same buffer conditions. The mobility of a striped particle is described as

$$
v_{E}=\frac{\varepsilon_{r} \varepsilon_{0}}{\eta} \int_{-1}^{1} \zeta(z) d z
$$

where $\zeta(z)$ is the nonuniform zeta potential along the particle's length [Fair and Anderson, Rose]. The axial coordinate, $z$, is non-dimensionalized by the particle halflength. For a particle with two stripe materials and uniform stripe lengths the mobility is simply

$$
v_{E}=\frac{\varepsilon_{r} \varepsilon_{0}}{\eta}\left(\frac{\zeta_{1}(\# \text { metal } 1 \text { stripes })}{\text { total \# stripes }}+\frac{\zeta_{2}(\# \text { metal } 2 \text { stripes })}{\text { total \# stripes }}\right)
$$

where $\zeta_{1}$ and $\zeta_{2}$ are the zeta potentials of each metal. The mobility is only a function of the total amount of material for each stripe type and not the actual striping pattern. For the particles in this study, quadrupole moments due to the striping generally contribute less than $1 \%$ to the net mobility and are therefore neglected [Fair] and [Rose].

\section{Materials and Methods}


Particles were synthesized by electrodeposition in porous anodic alumina templates [33, 34] using the procedure described in [20]. Particles were approximately $250 \mathrm{~nm}$ in diameter and $6 \mu \mathrm{m}$ long. Single-material Au, Ag, and Pd particles as well as Au/Ag striped particles were synthesized. Upon dissolution of the alumina template material in $3 \mathrm{M} \mathrm{NaOH}$, particles were pelleted and resuspended three times in either deionized water (in the case of bare, uncoated particles) or an aqueous solution of 20\% 11mercaptoundecanoic acid (MUA), which spontaneously forms a thiol-linked selfassembled monolayer (SAM) coating on the metal surface. In addition, some particles were subsequently derivatized with proteins by attaching them to the carboxyl groups at the free ends of the MUA molecules using the process based on 1-ethyl-3-(3dimethylaminopropyl) carbodiimide hydrochloride (EDP) and sulfo-Nhydroxysuccinimide [35]. The proteins used included ovalbumin (Ova, Sigma, St. Louis, MO) and rabbit anti-Ova antibody (American Type Culture Collection, Manassas, VA). In addition, carboxylated 5.26 micron polystyrene beads (Spherotech, Libertyville, IL) were also tested for comparison and calibration purposes. The surface functionalization states of the particles prepared for this study are diagrammed in Fig. 2.

In addition, additional batches of Au particles were synthesized and coated by similar means with self-assembled monolayers of two alternative thiol molecules, mercaptoethanol (ME) or mercaptoethanesulfonic acid (MESA).

Zeta potential measurements were performed using a ZetaPLUS zeta potential measurement system (Brookhaven Instruments, Holtsville, NY). This system employs 
the electrophoretic light scattering method to measure the electrophoretic velocities of suspended particles in an applied electric field, yielding the zeta potential.

Particles were suspended in saline solutions prepared by adding reagent-grade $\mathrm{NaCl}$ to deionized water. The salt content was used to fix the ionic strength so that the addition of dilute acid or base to adjust the $\mathrm{pH}$ would not significantly affect the overall conductivity of the solution. Solutions of $1 \mathrm{mM}$ and $10 \mathrm{mM} \mathrm{NaCl}$ were prepared. Higher concentrations result in a solution too conductive to be analyzed using this method.

The $\mathrm{pH}$ of the solutions was varied by starting with a suspension of particles in pure saline solution, and then adding small amounts of either dilute $\mathrm{HCl}$ or dilute $\mathrm{NaOH}$ to adjust the $\mathrm{pH}$ between measurements. The choice of acid and base was made to simplify the ionic content of the solution as much as possible by limiting the dissolved species to only the monovalent ions $\mathrm{Na}^{+}$and $\mathrm{Cl}^{-}$, along with $\mathrm{H}^{+}$and $\mathrm{OH}^{-}$ions. Measurements of $\mathrm{pH}$ were taken using an electronic $\mathrm{pH}$ meter (Cole-Parmer, Vernon Hills, IL) prior to the zeta potential measurements at each new condition. The $\mathrm{pH}$ of individual samples was varied in one direction (from neutral toward either low or high $\mathrm{pH}$ ), in order to avoid the addition of acid and base to the same sample, which would have altered the saline concentration. The concentration (number density) of particles was approximately $2 \times 10^{7} / \mathrm{ml}$ in all cases.

The ZetaPLUS performs a set of ten individual tests at each condition and averages the results to provide a final value for the zeta potential. In order to verify repeatability, two 
to five such sets were taken and the results averaged to arrive at the final zeta potential value for each condition. The data for each particle type at each saline concentration includes the measurements for at least two independent batches of particles, to verify repeatability. The applied field for each experiment was between 10 and $15 \mathrm{~V} / \mathrm{cm}$.

\section{Results and Discussion}

3.1 Monometallic particles and general observations

Zeta potential data for monometallic Au, Ag, and Pd particles with and without MUA coatings are shown in Fig. 3a-f. The data are plotted against the $\mathrm{pH}$ of the saline solution, and the plots include comparative data series for solutions at $1 \mathrm{mM}$ and $10 \mathrm{mM} \mathrm{NaCl}$ concentration.

These results exhibit some general trends that were observed across all the zeta potential tests. The zeta potentials are, in essentially all cases, negative. The absolute value of the zeta potential is typically maximized at $\mathrm{pH}$ values in the neutral or slightly basic range, and falls off toward the acidic and basic extremes. This is consistent with earlier results reported for thiolate-modified gold particles [36]. The results show that the drop is more pronounced toward the acid end of the range, where the values of the zeta potential approach zero. The general shape of the observed zeta potential vs. pH behavior, particularly in the acidic range, is similar to that observed for glass and PDMS silicone polymer, two materials commonly used in the construction of microfluidic devices [37- 
43]. Results of experiments on these materials in the literature show values ranging from -40 to $-90 \mathrm{mV}$ at moderate to high $\mathrm{pH}$, trending in an almost linear fashion toward zero near $\mathrm{pH}$ 2. This suggests that both particle-particle and particle-wall aggregation will be greatest in low $\mathrm{pH}$ solutions.

While performing the tests it was observed that the measurements were less stable and reproducible in the $1 \mathrm{mM}$ solution, and at $\mathrm{pH}$ values near neutral. This variability was reduced for the MUA-coated particles. This is consistent with earlier reports [36] suggesting greater sensitivity of the system to background contaminant species at low ionic strength conditions. The SAM coating may provide a more chemically uniform surface that is less sensitive to contamination, by either byproducts of the synthesis reaction or impurity species in the solution.

During tests of Ag-containing particles at low $\mathrm{pH}$ values, the measured zeta potential would often become more negative during the course of the measurement. The more acidic the solution, the more pronounced this effect became, and it was more pronounced with bare particles than with MUA-coated particles. This instability of the surface chemistry was most likely due to the time-dependent reaction of $\mathrm{HCl}$ with metallic Ag to form a $\mathrm{AgCl}$ layer.

With specific reference to Fig. 3, the data for the various bare metallic particles appear similar, regardless of material. The maximum zeta potentials observed are in the -30 to $50 \mathrm{mV}$ range. Bare particles also do not display much change in behavior between the 
two values of ionic strength. Theoretical studies suggest that for most of its range the zeta potential should vary as the negative log of the counterion concentration in M [42], but the effect was not clearly observable between the results for 1 and $10 \mathrm{mM}$ solutions. The comparison results for carboxylated polystyrene beads, shown in Fig. 4, also show minimal observed difference in zeta potential between the two ionic strength conditions.

The addition of the MUA self assembled monolayer coating had little observable effect on the electrokinetic properties of the Pd and Ag particles, but a dramatic effect in the case of Au particles. At an ionic strength of $10 \mathrm{mM}$ (Fig. 3b), the zeta potential for the MUA-coated Au particles can be more than twice that of the uncoated particles. A similar, but much less dramatic, increase can be seen at $1 \mathrm{mM}$ (Fig. 3a). Figure 3b indicates that the zeta potential data for the coated and uncoated particles are similar at very low $\mathrm{pH}$, but diverge at around $\mathrm{pH} 3$. It is likely that this divergence is due to the titration of the carboxylic acid end groups of the MUA molecules, which, upon the dissociation of $\mathrm{H}^{+}$ions, adds a layer of additional negative charge to the particles. The use of SAM coatings with different titratable end groups would be expected to produce changes in the zeta potential corresponding to the pKa values of the respective end groups. 
It is possible that the formation of the thiol-linked MUA coating is not as efficient on the Ag and Pd surfaces as on Au. While elementary hard-soft acid-base theory [44,45] suggests that thiol linkages should form with all these metals, Au surfaces are more chemically inert than the others. During the release of particles from the alumina template and subsequent washing, a few monolayers of oxide may form on the surfaces of the Ag and Pd particles, which could have the effect of inhibiting the attachment of a dense SAM coating.

\subsection{Alternative SAM coatings}

Comparative data for Au particles with each of the SAM coatings is given in Fig. 5a, showing the relative effect of the different coating chemistries. All of the chosen molecules possess identical thiol linkages and carboxyl-terminated free ends, their differences being in the intervening hydrocarbon backbone. All of the SAM coatings show a qualitatively similar zeta potential enhancement effect, but the magnitude of the effect is greatest for the MUA coating. The zeta potential model in Eq. (8) is applied to the curves to determine the total number of surface groups, pKa, and charge layer capacitance for each coating type. The theoretical curves are superimposed in Fig. 5b, and the resulting parameter data is shown in Table 1 below. The difference in magnitude in zeta potential between the SAM types appears to be primarily due to the difference in the number of surface groups which corresponds to packing density. The isoelectric point for the particle with a given polymer coating is directly related to the pKa of the polymer coating. 


\subsection{Bimetallic particles}

Data for Au, Ag, and Au/Ag striped particles with MUA coatings at a constant $10 \mathrm{mM}$ ionic strength are shown in Fig. 6, demonstrating the effect of particle composition. The total lengths of the Au and Ag segments in the striped particles were equal. The net zeta potential for the $\mathrm{Au} / \mathrm{Ag}$ particles, predicted using Eq. (5), is $\zeta_{A g / A u}=\left(\zeta_{A g}+\zeta_{A u}\right) / 2$. The Au and Ag values are determined from the measured values for the homogenous Ag and Au particles. This predicted behavior is shown with a dotted line in Fig. 6, and agrees well with the measured $\mathrm{Ag} / \mathrm{Au}$ data across the $\mathrm{pH}$ range.

\subsection{Protein-functionalized particles}

The data for Au particles that have been MUA coated and subsequently derivatized with protein molecules are shown in Fig. 7. These measurements were taken in $10 \mathrm{mM} \mathrm{NaCl}$ solution. Here, the absolute values of zeta potential are in a favorable $-30 \mathrm{mV}$ or greater range, but the maximum otherwise seen near neutral conditions is suppressed in favor of a flatter and more uniform trend with $\mathrm{pH}$. The less distinct behavior may be attributed to the complex chemistry of the large ovalbumin $(45 \mathrm{kD})$ and immunoglobulin $\mathrm{G}(146 \mathrm{kD})$ molecules, each of which possess a large number of titratable residues of different types. The scatter in this data is much greater than seen in the prior tests, a result that may be attributed to variation in the density of protein conjugation, particularly between different batches. 
As noted above, the addition of MUA coating, at least on Au particles, led to an enhancement in zeta potential. When such coating was subsequently derivatized with proteins this effect was suppressed. The zeta potential of the protein-coated particles is somewhat buffered against the effects of $\mathrm{pH}$, and do not exhibit the sharp deviation from bare particle behavior in the weak acid range seen with MUA coated particles.

Ovalbumin protein possesses a known isoelectric point $\mathrm{pI}=4.6$ [46], and immunoglobulins typically possess isoelectric points in the 5-7 range, but the sharpness of the charge transition is expected to be much less than for a particle exhibiting a single species of titratable acid residue.

\section{Concluding Remarks}

In this work, we have studied the zeta potential of metallic nanowire particles in aqueous saline solution as a function of $\mathrm{pH}$, ionic strength, metallic composition, and surface functionalization state. The results are useful in predicting and controlling the aggregation, adhesion, and transport of such particles within microfluidic devices and other systems.

While further work will be useful in understanding the different aspects of behavior seen, a few practical conclusions can be drawn. In all cases, the magnitude of the zeta potential is greatest at moderate $\mathrm{pH}$ and least at very low $\mathrm{pH}$; in order to minimize aggregation and adhesion to glass or PDMS surfaces, or to maximize electrokinetic mobility, acidic 
solutions should be avoided. Ionic strength effects are not pronounced for these particles within the limited range (1-10 mM) used in the study. Thiolated SAM coatings can have a dramatic effect on the zeta potential of the metallic particles, though the effect is much more evident for Au particles than for particles of less noble metals, which may be less ideal for the attachment of dense thiolated coatings. The zeta potential enhancement appears to be due to the dissociation of titratable end groups, and is more pronounced at the higher ionic strength conditions corresponding to a thinner ionic double layer.

This work was performed under the auspices of the U.S. Department of Energy by the University of California, Lawrence Livermore National Laboratory under Contract W7405-Eng-48, with funding from the Laboratory Directed Research and Development Program. 


\section{References}

[1] Leuvering, J. H. W., Thal, P. H. J. M., van der Waart, M., Schuurs, A. H. W. M., J. Immnunol. Meth. 1981, 45, 183-194.

[2] Hayat, M. A., (Ed.), Colloidal Gold: Principles, Methods and Applications, Academic Press, San Diego, 1989.

[3] Elghanian, R., Storhoff, J. J., Mucic, R. C., Letsinger, R. L., Mirkin, C. A., Science 1997, 277, 1078-1081.

[4] Taton, T. A., Mirkin, C. A., Letsinger, R. L., Science 2000, 289, 1757-1760.

[5] Cao, Y. C., Jin, R., Mirkin, C. A., Science 2002, 297, 1536-1540. (also Raman)

[6] Nam, J.-M., Thaxton, C. S., Mirkin, C. A., Science 2003, 301, 1884-1886.

[7] Pileth, W. J., J. Phys. Chem. 1982, 86, 3166-3170.

[8] Freeman, R. G., Grabar, K. C., Allison, K. J., Bright, R. M., Davis, J. A., Guthrie, A. P., Hommer, M. B., Jackson, M. A., Smith, P. C., Walter, D. G., Natan, M. J., Science 1995, 267, 1629-1632.

[9] Mulvaney, P., Langmuir 1996, 12, 788-800.

[10] Nie, S., Emory, S. R., Science 1997, 275, 1102-1106.

[11] Lyon, L. A., Musick, M. D., Smith, P. C., Reiss, B. D., Pena, D. J., Natan, M. J., Sensors and Actuators B 1999, B54, 118-24.

[12] Dou, X., Jung, Y. M., Yamamoto, H., Doi, S., Ozaki, Y., Appl. Spectrosc. 1999, 53, 133-138.

[13] Horisberger, M., Rosset, J., Bauer, H., Experientia 1975, 31, 1147-1149.

[14] Geoghegan, W. D., Ackerman, G. A., J. Histochem. Cytochem., 1977, 25, $1187-$ 1200.

[15] Tkachenko, A. G., Xie, H., Coleman, D., Glomm, W., Ryan, J., Anderson, M. F., Franzen, S., Feldhein, D. L., J. Am. Chem. Soc. 2003, 125, 4700-4701. 
[16] Mirkin, C. A., Letsinger, R. L., Mucic, R. C., Storhoff, J. J., Nature 1996, 382, 607609.

[17] Alivisatos, A. P., Johnsson, K. P., Peng, X., Wilson, T. E., Loweth. C. J., Bruchez, M. P., Schultz, P. G., Nature 1996, 382, 609-611.

[18] Martin, B. R., Dermody, D. J., Reiss, B. D., Fang, M., Lyon, L. A., Natan, M. J., Mallouk, T. E., Adv. Mater. 1999, 11, 1021-1025.

[19] McConnell, W. P., Novak, J. P., Brousseau, L.C., Fuierer, R. R., Tenent, R. C., Feldheim, D. L., J. Phys. Chem. B 2000, 104, 8925-8930.

[20] Nicewarner-Pena, S. R., Freeman, R. G., Reiss, B.D., He, L., Pena, D. J., Walton, I. D., Cromer, R., Keating, C. D., Natan, M. J., Science 2001, 294, 137-141.

[21] Tanase, M., Bauer, L. A., Hultgren, A., Silevitch, D. M., Sun, L., Reich, D. H., Searson, P. C., Meyer, G. J., Nano Letters 2001, 1, 155-158.

[22] Walton, I. D., Norton, S. M., Balasingham, A., He, L., Oviso, D. F., Gupta, D., Raju, P. A., Natan, M. J., Freeman, R. G., Anal. Chem. 2002, 74, 2240-2247.

[23] Keating, C. D., Natan, M. J., Adv. Mater. 2003, 15, 451-454.

[24] Reich, D. H., Tanase, M., Hultgren, A., Bauer, L. A., Chen, C. S., Meyer, G. J. J. Appl. Phys. 2003, 93, 7275-7280.

[25] Lee, K.-B., Park, S., Mirkin, C. A., Angew.Chem. Int. Ed. 2004, 43, 3048-3050.

[26] Davison, S. M., Mayer, T. S., Sharp, K. V., in: Proc. 2004 ASME Intl. Mech. Eng. Cong. and Exhibition, ASME, 2004, \#IMECE2004-60598, 1-6.

[27] Deryaguin, B. V., Landau, L., Acta Physiochim. USSR 1941, 14, 633-662.

[28] Verwey, E. J. W., Overbeek, J. T. G., Theory of Stability of Lyophobic Colloids, Elsevier, Amsterdam, 1948.

[29] von Smoluchowski, M., in: Graetz (Ed.), Handbuch der Electrizitat und des Magnetismus, Vol II, Barth, Leipzig, 1921.

[30] Hunter, R. J., Zeta Potential in Colloid Science, Academic Press, San Diego, 1981.

[31] Overbeek, J. in: Kruyt (Ed.), Colloid Science, Elsevier, Amsterdam and London, 1952, 207-213.

[32] Simonov, I. N., Dukhin, S. S., Colloid J. of the USSR 1973, 35, 173-176. 
[33] Al-Malawi, D., Liu, C. Z., Moskovits, M., J. Mater. Res. 1994, 9, 1014-1018.

[34] Hulteen, J. C., Martin, C. R., J. Mater. Chem. 1997, 7, 1075-1087.

[35] Johnsson, B., Löfas, S., Lindquist, G., Anal. Biochem. 1991, 198, 268-277.

[36] Kimura, K., Takashima, S., Ohshima, H., J. Phys. Chem B 2002, 106, 7260-7266.

[37] Ocvirk, G., Munroe, M., Tang, T., Oleschuk, R., Westra, K., Harrison, D. J., Electrophoresis 2000, 21, 107-115.

[38] Buch, J. S., Wang, P.-C., DeVoe, D. L., Lee, C. S., Electrophoresis 2001, 22, 39023907.

[39] Gu, Y., Li, D., J. Coll. Interf. Sci. 2000, 226, 328-339.

[40] Park, J.-G., Lee, S.-H., Kim, H.-G., in Chemical-Mechanical Polishing:

Fundamentals and Challenges, Babu, S.V., Danyluk, S., Krishnan, M. I., Tsujimura, M. (Eds.), Mat. Res. Soc., Vol. 566, 2000.

[41] Spehar, A.-M., Koster, S., Linder, V., Kulmala, S., do Rooij, N. F., Verpoorte, E., Sigrist, H., Thormann, W., Electrophoresis 2003, 24, 3674-3678.

[42] Kirby, B. J., Hasselbrink, E. F. Electrophoresis 2004, 25, 187-202.

[43] Kirby, B. J., Hasselbrink, E. F. Electrophoresis 2004, 25, 203-213.

[44] Pearson, R.G., J. Am. Chem. Soc. 1963, 85, 3533-3543.

[45] Swaddle, T. W., Inorganic Chemistry: An Industrial and Environmental Perspective, Academic Press, San Diego, 1997.

[46] Holen E, Elsayed S., Int. Arch. of Allergy and Appl. Immunol. 1990, 91, 136-141.

M.C. Fair and J.L. Anderson, J. Colloid Interf. Sci. 127, 388 (1989).

Rose, K.A., J.A. Meier, G.M. Dougherty, and J.G. Santiago, Rotational Electrophoresis of Striped Metallic Microrods. Physical Review E, 2007. 75: p. 011503. 


\section{Figures}

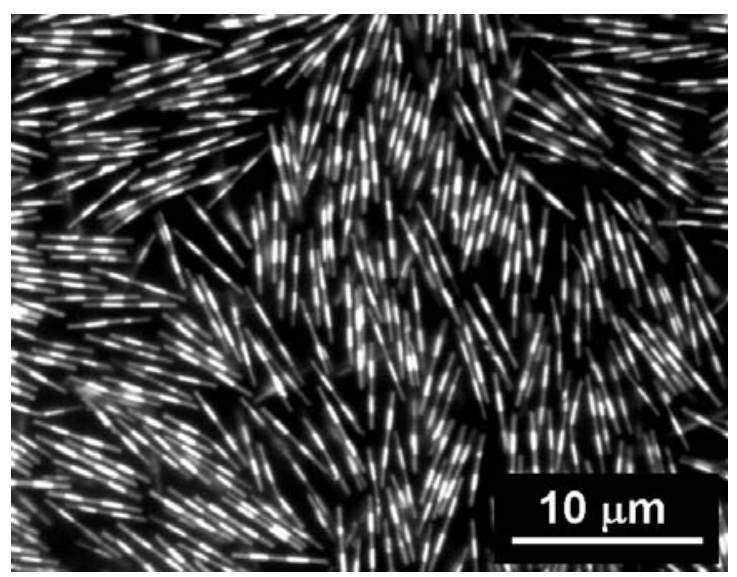

Figure 1. Optical reflectance microscopy image of striped Au/Ag Nanobarcodes ${ }^{\circledR}$ particles. Most of the particles tested were single-metal particles of the same dimensions.

a)

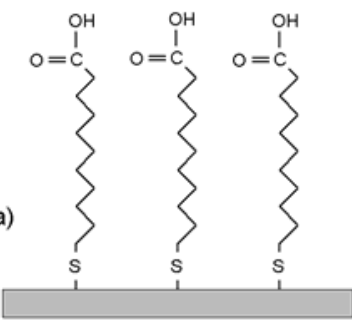

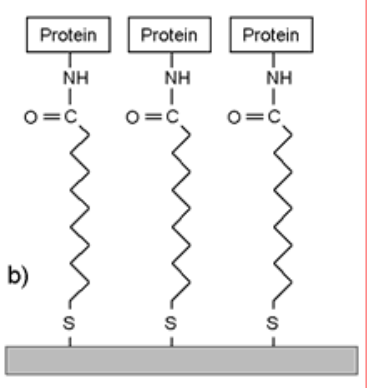

Figure 2. Diagram of the surface functionalization conditions of the particles tested. In addition to bare metal surface, these included: a) thiol-linked mercaptoundecanoic acid (MUA, shown) or alternate self-assembled alkanethiol monolayer; and b) MUA with proteins conjugated at the terminal carboxyl residues.

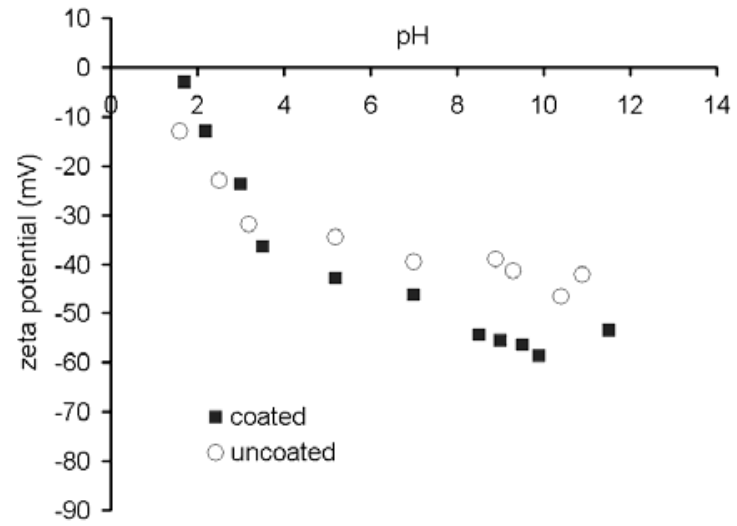

Fig. 3(a). 


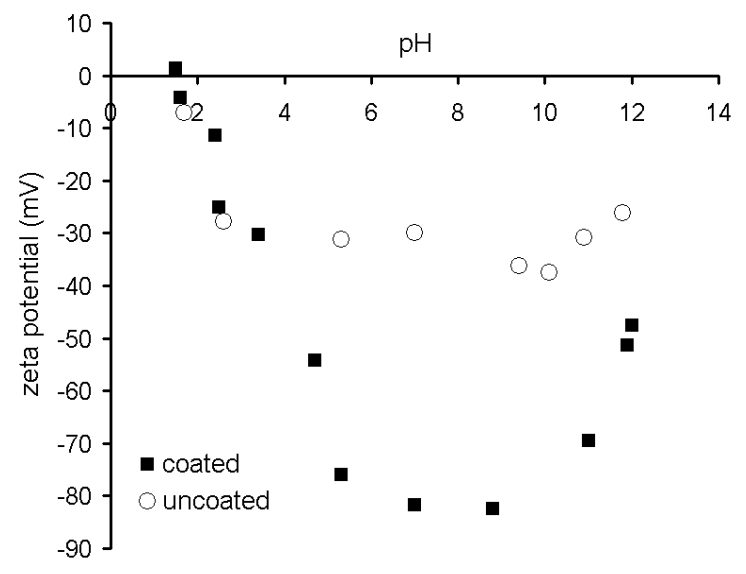

Fig. 3(b).

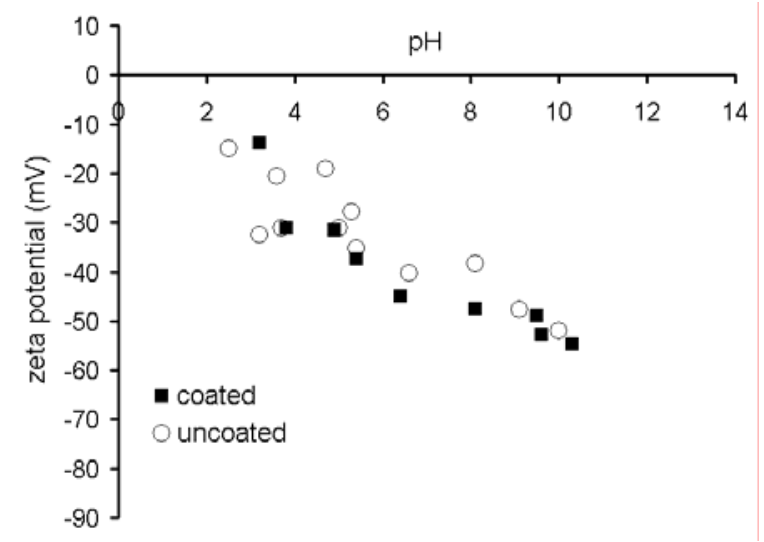

Fig. 3(c).

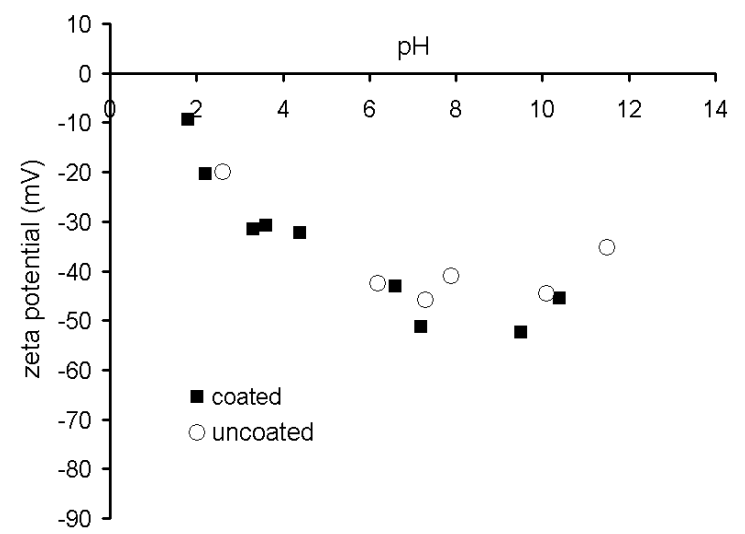

Fig. 3(d). 


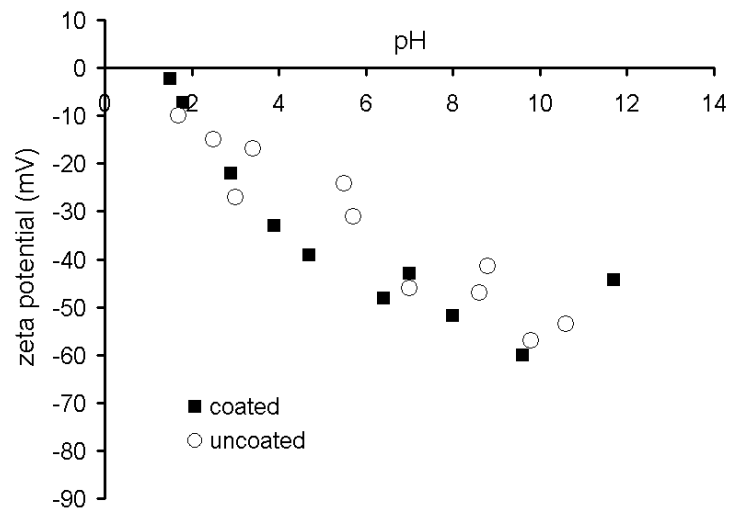

Fig 3(e).

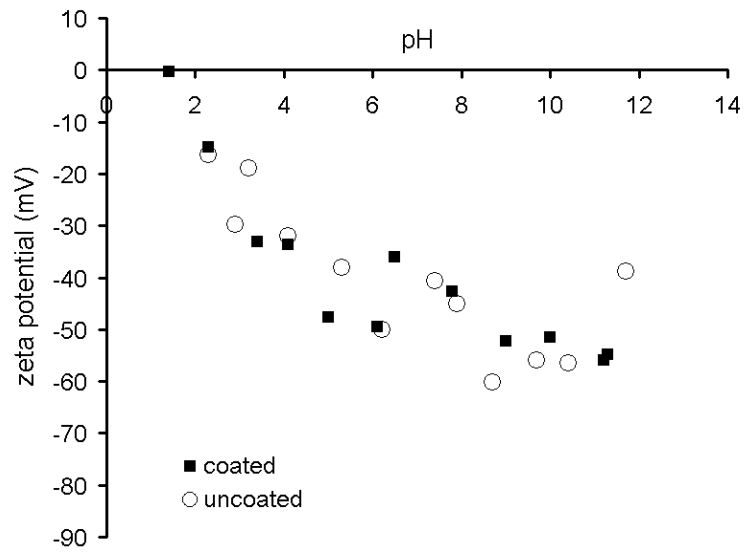

Fig. 3(f).

Figure. 3(a)-(f). Zeta potential versus $\mathrm{pH}$ data for MUA-coated and uncoated monometallic nanorod particles in $\mathrm{NaCl}$ solutions of different ionic strengths: (a) Au particles at $1 \mathrm{mM}$; (b) Au particles at $10 \mathrm{mM}$; (c) Ag particles at $1 \mathrm{mM}$; (d) Ag particles at $10 \mathrm{mM}$; (e) Pd particles at $1 \mathrm{mM}$; (f) Pd particles at $10 \mathrm{mM}$.

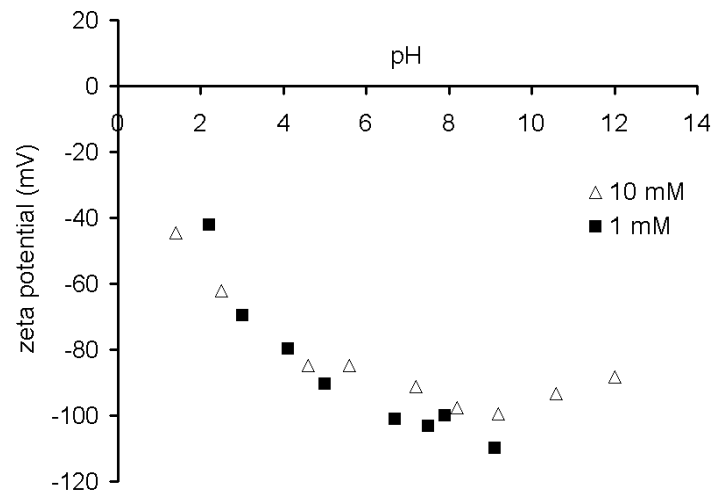

Figure 4. Data for polystyrene particles in $1 \mathrm{mM}$ and $10 \mathrm{mM} \mathrm{NaCl}$ 

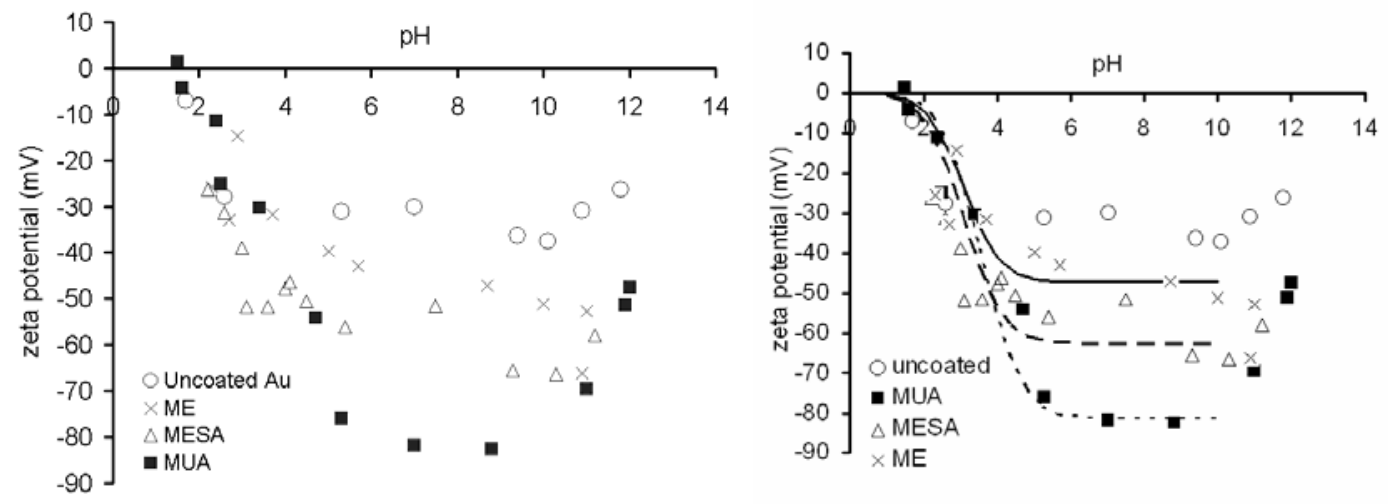

Figure 5. Comparative data for Au particles in $10 \mathrm{mM} \mathrm{NaCl}$ with four alternative SAM coatings: (a) zeta potential data alone; (b) data with theoretical curves for coated particles from Eq. (8) superimposed. The solid line refers to ME, the dashed to MESA, and the dotted to MUA.

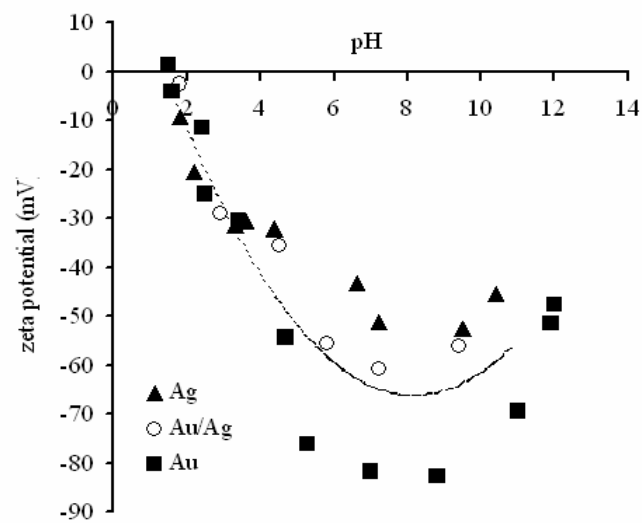

Figure 6. Comparative data for MUA-coated Ag, Au, and Au/Ag striped particles in 10 $\mathrm{mM} \mathrm{NaCl}$, with the theoretical prediction for 50\% Au / 50\% Ag striped particles indicated by the dotted line. 


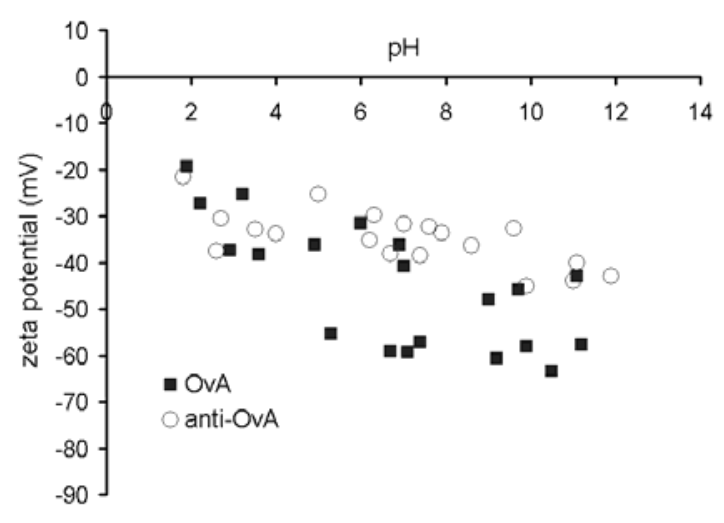

Figure 7. Data for Au particles in $10 \mathrm{mM} \mathrm{NaCl}$, conjugated with ovalbumin or antiovalbumin antibody proteins.

\section{Table}

Table 1. Parameter values for the various SAM coatings, used to generate the theoretical curves in Fig. 5b using Eq (8).

$\begin{array}{cccc} & \text { MUA } & \text { MESA } & \text { ME } \\ N_{s} & & & \\ \left(\text { sites } / \mathrm{m}^{2}\right) & 1.8 \times 10^{17} & 1.3 \times 10^{17} & 9.0 \times 10^{16} \\ \mathrm{pKa} & 2.3 & 2.0 & 3.4 \\ \mathrm{~K} & & & \\ \left(\mathrm{~F} / \mathrm{m}^{2}\right) & 20 \times 10^{-2} & 26 \times 10^{-2} & 26 \times 10^{-2}\end{array}$

